\section{Ma. Mercedes Jiménez Narváez}

Universidad de Antioquia

Adriana Morales Perlaza

Université de Montréal

\title{
La formation pratique des enseignants en Colombie
}

\section{Chronique internationale}

\section{Introduction}

Dans son document Lineamientos decalidadpara las licenciaturas en educación (2014) ${ }^{1}$, le ministère de l'Éducation nationale de Colombie a exprimé le besoin qui existe actuellement dans le pays de revoir la qualité des programmes de formation initiale et continue des enseignants, ainsi que d'établir des paramètres de référence plus exigeants dans l'autorisation et le renouvellement de l'approbation des programmes. Ainsi, dans les deux prochaines années, plus de 300 baccalauréats en éducation devront amorcer un processus de renouvellement de leur approbation selon les nouvelles directives exprimées dans le document.

En ce qui a trait à la formation pratique, les directives actuelles du ministère exigent : a) une durée de 5 ans pour tous les baccalauréats dont la formation pratique prévaut comme axe central de la formation; b) des stages intégrés dans les programmes à partir du troisième semestre, avec des activités d'observation de la part des stagiaires, les stages augmentant de façon exponentielle pour devenir l'axe principal de la formation; c) que les stages doivent être surveillés et

1 En français : Lignes directrices de la qualité dans les baccalauréats en éducation. En ce qui a trait au système de formation des enseignants, le ministère de l'Éducation a proposé la division de celui-ci en trois sous-systèmes : la formation initiale, la formation continue et la formation avancée. Le document de 2014 présente les principales caractéristiques de ces trois sous-systèmes de formation, de même que les directives pour leur évaluation et leur vérification. Cela dit, ce document est encore en processus de discussion et de construction. 
accompagnés par des professeurs spécifiquement désignés à cet effet; d) que les programmes peuvent ajuster la longueur de la formation pratique si leur but est un double diplôme ou un passage vers le programme de maîtrise; e) de fournir des environnements d'apprentissage pour le développement des stages à partir de la deuxième année du programme; f) de donner une priorité à la recherche sur les pratiques pédagogiques pour atteindre la qualité, l'articulation et la pertinence de la diversité des environnements d'apprentissage; g) que tous les programmes de premier cycle devront s'offrir en présentiel compte tenu de la nature et l'importance de la pratique.

Face à ces nouvelles régulations, quel est le panorama actuel de la formation pratique des enseignants en Colombie et quels changements devront être apportés par les universités à leur offre de formation initiale à l'enseignement? Dans ce chapitre, nous exposons, dans un premier temps, le système éducatif ainsi que la formation initiale des enseignants en Colombie. Dans un deuxième temps, nous présentons la formation pratique dans le cadre de leur formation initiale en analysant un cas particulier : la Faculté d'éducation de l'Université d'Antioquia. Nous aborderons finalement les défis actuels face à ce contexte de renouvellement des structures de formation, incluant notamment le renouvellement de la formation pratique (stages) dans les baccalauréats en éducation.

\section{Le système éducatif et la formation des enseignants en Colombie}

En Colombie, le système éducatif est administré par le ministère de l'Éducation nationale dont la direction générale se situe à Bogota, la capitale du pays. Les départements et villes fonctionnent de manière décentralisée à travers les Secrétaires de l'éducation et, dans chaque ville, des écoles privées et publiques offrent l'enseignement à tous les niveaux de la maternelle jusqu'à la $11^{\mathrm{e}}$ année (voir la Figure 1).

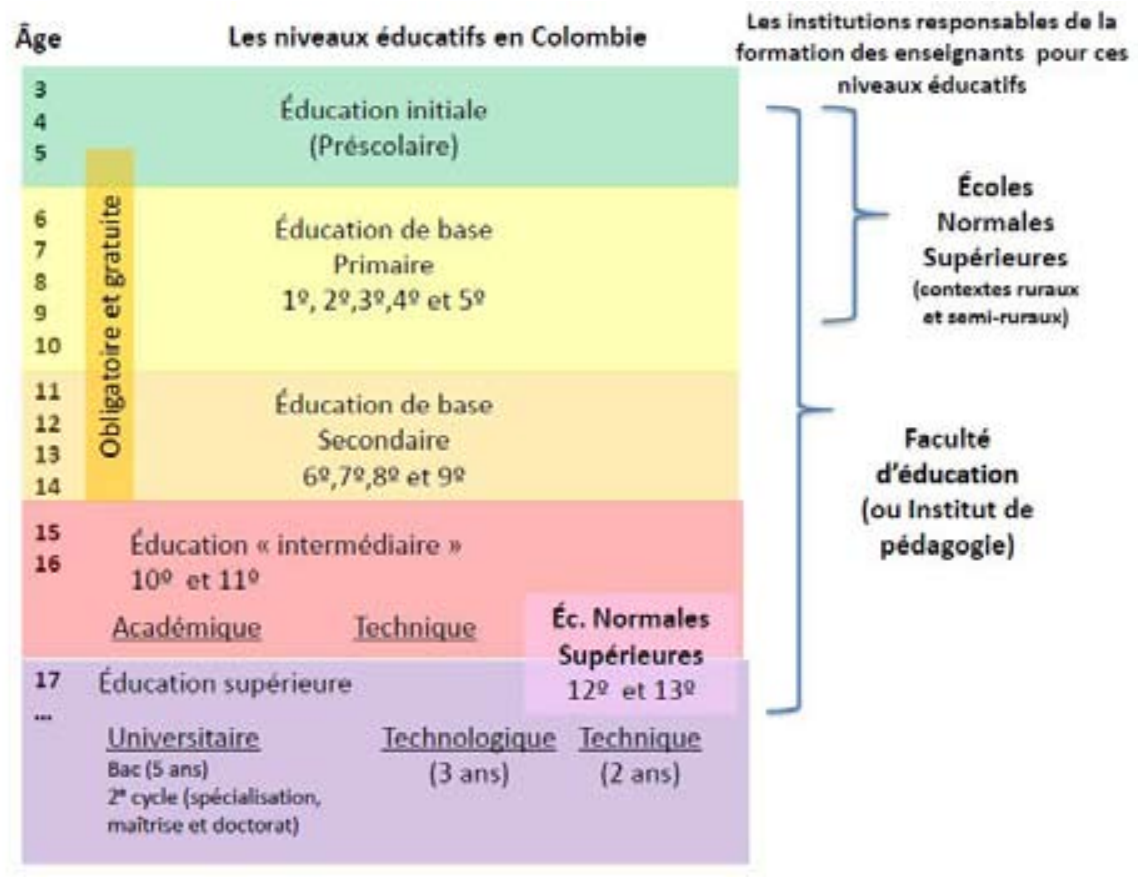

Figure 1

Les niveaux éducatifs et la formation des enseignants en Colombie. 
La formation des enseignants en Colombie se réalise dans des Écoles normales supérieures (ENS) pour les enseignants de l'école maternelle et primaire dans des contextes ruraux et semi-ruraux; et dans l'université, dans les Facultés ou Instituts d'éducation, pour les enseignants de l'école maternelle, primaire, secondaire et pour l'éducation supérieure ( $1^{\text {er }}$ et $2^{\text {e }}$ cycle; formation continue) dans les contextes urbains.

En ce qui a trait à la formation universitaire, celle-ci se réalise selon les domaines de contenus prévus par la Loi générale de l'éducation ${ }^{2}$ qui se subdivisent ainsi : 1) sciences de la nature et éducation à l'environnement; 2) sciences sociales, histoire, géographie, constitution politique et démocratie; 3) éducation artistique; 4) éducation éthique et valeurs humaines; 5) éducation physique, récréation et sport; 6) éducation religieuse; 7) humanités, espagnol et langues étrangères; 8) mathématiques; 9) technologies et informatique.

Étant donné que chaque faculté d'éducation est autonome dans l'organisation des contenus des programmes, nous nous limitons, dans le cadre de cet article, à la présentation de la formation pratique des enseignants dans une faculté d'éducation en Colombie : celle de l'Université d'Antioquia à Medellín. Il existe dans cette faculté trois départements et huit programmes de formation, chacun d'une durée de 5 ans (10 semestres) :1) Département de l'enseignement des sciences et des arts : Baccalauréat en éducation de base, concentration mathématiques; Baccalauréat en mathématiques et physique; Baccalauréat en éducation de base, concentration sciences de la nature et éducation à l'environnement; Baccalauréat en éducation de base, concentration en sciences sociales; Baccalauréat en éducation de base, concentration en humanités et espagnol; 2) Département de l'éducation des enfants: Baccalauréat en pédagogie de l'enfance; Baccalauréat en éducation spécialisée; 3) Département de pédagogie : Baccalauréat en pédagogie de la Terre mère ${ }^{3}$.

Tous les programmes sont constitués de cours de pédagogie, de didactique et des connaissances spécifiques (disciplinaires). Depuis 2010, les baccalauréats ci-haut mentionnés partagent dans leur plan de formation un élément commun, celui d'être composé de 17 cours que les étudiants peuvent suivre en fonction de leurs intérêts et des horaires.

\section{La formation pratique}

Dans ces programmes de formation, la pratique pédagogique est une question transversale, avec des intensités différentes et dans des contextes différents selon les programmes. Elle est régie par un Règlement de pratique où sont définis les caractéristiques, buts, modalités, droits et devoirs des acteurs (étudiants, professeurs-conseillers, enseignants associés) ainsi que les institutions impliquées (universités et centres de pratique).

De manière générale, la pratique est conçue comme « un ensemble de relations théoriques et pratiques articulées aux différentes dimensions pédagogique, didactique, disciplinaire et de recherche.Cette relation est dynamique et complexe en plus d'être traversée par des référents éthiques, culturels et politiques où sont incluses des logiques sociales, institutionnelles et personnelles, qui permettent à l'enseignant

(Loi 115 de 1994)

3 Ce programme porte sur des modèles pédagogiques en contexte des peuples indigènes, liant communauté et éducation, nature, territoire, enjeux d'un système éducatif colonisateur vs indigène, éducation multiculturelle et interculturelle. 
en formation de se consolider, à partir d'une attitude critique et réfléchie, en tant qu'intellectuel de la pédagogie ${ }^{4}$ " (Accord 248 de 2012. Règlement de pratique de la Faculté d'éducation).

Dans cinq programmes (voir Tableau 1), les stages débutent dès les premiers semestres. Dans les autres programmes de la faculté, les stages ne se trouvent que dans les trois derniers semestres (stages de fin d'études). Les premiers stages dans ces cinq programmes sont réalisés pendant 40 heures par semaine et étalés sur 10 semaines ${ }^{5}$. Les stages de fin d'études se réalisent pendant les $8^{\mathrm{e}}$, $9^{\mathrm{e}}$ et $10^{\mathrm{e}}$ semestres des programmes, dont les intitulés de formation se déclinent respectivement ainsi : pratique pédagogique I, II et travail de fin d'études (mémoire de baccalauréat). Dans les stages de fin d'études, les étudiants passent en moyenne 10 heures par semaine pendant 18 semaines à réaliser leur formation pratique.

Tableau 1

Les stages dans cinq baccalauréats de la Faculté d'éducation, Université d'Antioquia.

\begin{tabular}{|c|c|c|c|c|c|c|c|}
\hline Programme & Semestre 1 & Semestre 2 & Semestre 3 & Semestre 4 & Semestre 5 & Semestre 6 & Semestre 7 \\
\hline $\begin{array}{l}\text { Pédagogie } \\
\text { de l'enfance }\end{array}$ & $\begin{array}{l}\text { Les mères } \\
\text { enceintes } \\
\text { et les pères }\end{array}$ & $\begin{array}{l}\text { Éducatrices } \\
24-60 \text { mois }\end{array}$ & $\begin{array}{c}\text { Mères } \\
\text { communautaires } \\
\text { résilience }\end{array}$ & $\begin{array}{l}\text { Développement } \\
\text { artistique }\end{array}$ & $\begin{array}{c}\text { Pensée } \\
\text { mathématique }\end{array}$ & $\begin{array}{c}\text { Pensée } \\
\text { scientifique }\end{array}$ & $\begin{array}{l}\text { École-famille et } \\
\text { communauté }\end{array}$ \\
\hline $\begin{array}{l}\text { Éducation } \\
\text { spécialisée }\end{array}$ & & $\begin{array}{l}\text { Contexte } \\
\text { scolaire }\end{array}$ & $\begin{array}{l}\text { Contextes non } \\
\text { scolaires }\end{array}$ & $\begin{array}{l}\text { L'enseignement } \\
\text { et les troubles } \\
\text { cognitifs }\end{array}$ & $\begin{array}{c}\text { L'enseignement } \\
\text { et les } \\
\text { handicaps } \\
\text { moteurs } \\
\end{array}$ & $\begin{array}{l}\text { L'enseignement } \\
\text { et la déficience } \\
\text { auditive }\end{array}$ & $\begin{array}{l}\text { L'enseignement } \\
\text { et la déficience } \\
\text { visuelle }\end{array}$ \\
\hline Espagnol & & & & Contextualisation & $\begin{array}{l}\text { Discours, } \\
\text { curriculum et } \\
\text { communauté }\end{array}$ & $\begin{array}{l}\text { École primaire } \\
\text { (de base) }\end{array}$ & $\begin{array}{c}\text { École } \\
\text { secondaire (de } \\
\text { base) }\end{array}$ \\
\hline $\begin{array}{l}\text { Sciences } \\
\text { sociales }\end{array}$ & & & & $\begin{array}{l}\text { Observation du } \\
\text { contexte scolaire }\end{array}$ & $\begin{array}{l}\text { Enseignement } \\
\text { en contexte } \\
\text { scolaire }\end{array}$ & $\begin{array}{c}\text { Projets } \\
\text { pédagogiques }\end{array}$ & $\begin{array}{l}\text { Enseignement } \\
\text { dans des } \\
\text { contextes non } \\
\text { scolaires }\end{array}$ \\
\hline $\begin{array}{l}\text { Pédagogie } \\
\text { de la Terre } \\
\text { mère }\end{array}$ & & & Communauté & Communauté & Communauté & Communauté & Communauté \\
\hline
\end{tabular}

$4 \quad$ Traduction libre.

5 Le semestre académique pour tous les cours est de 16 semaines, incluant les stages. 
Ainsi, le stage pratique pédagogique dure trois semestres consécutifs et est géré par le même professeur, nommé conseiller de pratique. Les étudiants participent à un séminaire hebdomadaire de 4 heures, stratégie privilégiée pour l'analyse, la réflexion critique et la confrontation des expériences que les étudiants construisent face aux composantes de l'enseignement, de la recherche et de la vulgarisation. Ils assistent de 8 à 10 heures par semaine aux centres de pratique qui peuvent être des contextes scolaires, des contextes non formels (ONG, fondations) ou des contextes dits non conventionnels (musées et parcs, hôpital, prison, bibliothèques, etc.).

Dans les séminaires, les étudiants et conseillers réalisent des lectures, présentent des textes et discutent de sujets à partir des points de vue pédagogique, didactique et de recherche; les étudiants réalisent un journal pédagogique (ou journal de bord) où ils enregistrent les expériences de la pratique; ils conçoivent et discutent des planifications des activités qui seront menées dans des centres de pratique et ils discutent aussi de ce qu'ils vivent dans leurs interventions en classe, de leurs progrès et difficultés. Dans les séminaires, ils réfléchissent sur leur rôle d'enseignants en général et de leur domaine en particulier (par exemple, les lectures de Freire sont source de réflexion et permettent de réaliser un lien entre la théorie et la pratique).

Dans le séminaire, on aborde aussi des sujets qui portent sur la recherche en éducation, ce qui rapproche les étudiants progressivement aux caractéristiques des paradigmes de recherche et du processus de construction d'un projet de recherche. Des équipes d'étudiants (2 ou 3) sélectionnent un thème, proposent des questions et des problèmes de recherche, identifient la route méthodologique qui est nécessaire selon les sujets de recherche choisis et interprètent, analysent et divulguent ce qu'ils ont trouvé. Le choix des objets d'étude doit être lié à des éléments didactico-pédagogiques et à des savoirs spécifiques qui portent sur l'activité enseignante; ils choisissent un groupe d'élèves ou une population avec lesquels ils réalisent leur intervention pédagogique et didactique tout en recueillant des données pour leur projet de recherche. La construction des protocoles d'éthique et des autorisations requises pour les projets sont aussi prises en considération. Ils peuvent également mener des études de recherche documentaire qui ne nécessitent pas d'intervention dans les contextes de pratique.

Le produit final est un rapport qui rend compte de la systématisation de l'expérience ou du processus de recherche avec des méthodologies existantes telles que: récit biographique, ethnographique, phénoménologique, recherche-action participative, études de cas, recherche expérimentale, quasi expérimentale ou ethnomathématique, etc. Certains des sujets de recherche ou des domaines qui ont été identifiés dans les programmes d'études de la Faculté d'éducation sont résumés dans le tableau 2 : 
Tableau 2

Sujets de recherche de mémoires de fin d'études selon le programme de formation.

\begin{tabular}{|c|c|}
\hline Programme & Sujets de recherche \\
\hline $\begin{array}{l}\text { Éducation de base, concentration } \\
\text { en Sciences sociales }\end{array}$ & $\begin{array}{l}\text { Le contexte scolaire; la relation élève-enseignant; la subjectivité de l'enseignant; les } \\
\text { politiques publiques; la citoyenneté. }\end{array}$ \\
\hline $\begin{array}{l}\text { Éducation de base, concentration } \\
\text { Sciences de la nature et Éducation } \\
\text { à l'environnement }\end{array}$ & $\begin{array}{l}\text { L'évaluation des apprentissages; I'histoire et l'épistémologie des sciences; l'éducation à } \\
\text { l'environnement; les pratiques expérimentales; les TIC et l'enseignement des sciences; le } \\
\text { rôle de la langue et de l'argumentation en classe de sciences; la formation des enseignants } \\
\text { des sciences. }\end{array}$ \\
\hline $\begin{array}{l}\text { Éducation de base, concentration } \\
\text { Mathématiques }\end{array}$ & $\begin{array}{l}\text { Le curriculum de mathématiques; les situations problèmes; la combinatoire, la } \\
\text { proportionnalité; la pensée spatiale; la pensée numérique; les objets virtuels } \\
\text { d'apprentissage. }\end{array}$ \\
\hline Mathématiques et Physique & $\begin{array}{l}\text { Enseignement et apprentissage des mathématiques et de la physique; formation pour la } \\
\text { civilité; argumentation; la relation de la physique et de l'art; l'activité expérimentale; le } \\
\text { programme de formation des enseignants; l'enseignement et l'inclusion. }\end{array}$ \\
\hline $\begin{array}{l}\text { Éducation de base, concentration } \\
\text { en Humanités et Espagnol }\end{array}$ & $\begin{array}{l}\text { L'enseignement de la langue et de la littérature; la lecture, l'écriture et les TIC; la littérature } \\
\text { concernant les arts; la lecture et l'écriture dans des contextes ruraux; l'oralité; la lecture et } \\
\text { l'écriture au niveau universitaire. }\end{array}$ \\
\hline Éducation spécialisée & $\begin{array}{l}\text { L'inclusion; l'intimidation à l'école; les difficultés d'apprentissage; la population autiste; les } \\
\text { outils multimédias. }\end{array}$ \\
\hline Pédagogie de l'enfance & L'enfance et le genre; I'enseignement des langues et TIC; contextes scolaires. \\
\hline
\end{tabular}

À la fin de chaque semestre, des journées de socialisation du projet sont réalisées (début des stages du $8^{\mathrm{e}}$ et $9^{\mathrm{e}}$ semestres) ainsi que des soutenances de mémoires (10 e semestre). Également, ces mémoires de baccalauréat sont évalués par un professeur externe qui fait des commentaires et des suggestions pour la qualification. Les mémoires de fin de programme peuvent être trouvés sur le site du Centre de documentation (CEDED) de la faculté d'éducation ${ }^{6}$.

\section{Les défis}

Les pratiques pédagogiques dans la faculté d'éducation de l'Université d'Antioquia sont l'une des principales composantes des plans de formation des différents programmes de baccalauréat. En effet, ces pratiques s'avèrent importantes dans la formation d'enseignants qui seront aussi des intellectuels de la pédagogie, avec des bases fortes en enseignement, recherche et vulgarisation. Cependant, puisque ce ne sont pas tous les baccalauréats qui commencent la formation pratique tôt dans la formation, ce sera l'un des principaux défis pour la nouvelle organisation des programmes de formation des enseignants, particulièrement dans les domaines des sciences de la nature et de l'environnement, et dans les programmes en mathématiques et physique.

Actuellement, et selon les nouvelles lignes directrices émises par le ministère de l'Éducation en 2014 , tous les programmes de formation initiale des enseignants doivent inclure une formation en langue seconde (anglais - 5 niveaux) et le début des pratiques à compter du troisième semestre. Ces

6 CEDED http://ayura.udea.edu.co/ceded/ 
lignes directrices impliquent que tous les baccalauréats doivent revoir leur structure pour intégrer ces composantes et que des décisions soient prises en ce qui a trait à l'inclusion des pratiques dès les premières années de la formation, incluant la définition des contenus à travailler, les contextes de pratique, les fonctions des conseillers et leur recrutement. Avant tout, il y aura besoin de générer une proposition qui favorise la réflexion sur la façon dont les stages seront articulés dans l'ensemble du programme de formation des enseignants. Ainsi, particulièrement dans le cas des programmes dont les stages sont effectués pendant les trois derniers semestres, avec une intensité de 6 heures par semaine, il semble que ce laps de temps (un an et demi) soit très faible par rapport aux besoins qu'ont les étudiants pour comprendre leur rôle en tant qu'enseignants. Par conséquent, la restructuration de la formation pratique doit également inclure une redistribution dans l'intensité horaire que les futurs enseignants peuvent avoir pour mieux connaître les contextes où ils vont travailler, leur permettant en même temps d'avoir assez de temps pour d'autres cours théoriques requis dans leur plan de formation.

Les expériences menées jusqu'à présent démontrent qu'il existe des difficultés inhérentes à la formation pratique, concernant tout particulièrement les relations avec les centres de pratique et avec les enseignants associés. Bien que diverses activités soient menées avec les établissements d'enseignement qui reçoivent les stagiaires (ils reçoivent des matériaux comme les règles de pratique et des accords de coopération sont établis à plusieurs reprises), dans plusieurs cas les enseignants associés laissent les stagiaires à eux-mêmes, délégant plus de responsabilités que celles convenues, changeant leurs horaires ou, tout à l'opposé, ne permettent aux stagiaires de participer à aucune activité. Ainsi, l'un des plus grands défis est de chercher des stratégies pour mieux resserrer les liens avec les centres de pratique et les enseignants associés, ces derniers n'ayant pas de bonification économique ou d'horaire, le désir d'accueillir un étudiant ou l'imposition de directeur d'école à assumer cette responsabilité étant les deux seuls facteurs les « motivant » à remplir un tel rôle.

Un dernier défi relève de l'articulation qui doit être opérée entre enseignement et recherche dans les pratiques pédagogiques, ainsi que le mémoire de baccalauréat à la suite de ces activités. La tâche d'enseignement combinée aux exigences de faire de la recherche formative implique pour les étudiants un certain nombre de difficultés liées aux exigences qui diffèrent dans ces deux activités parallèles. Alors que dans le stage ils réalisent des activités comme la planification, la conduite des classes, le contact avec d'autres enseignants, les parents, etc., pour effectuer leur projet de recherche, ils doivent réaliser d'autres activités telles que : la recherche d'informations, la proposition d'un problème, la conception et la mise en œuvre des instruments pour collecter des informations, etc. Bien qu'à travers les séminaires et la direction des mémoires il soit prévu que les étudiants articulent ces deux tâches, cela n'est pas toujours facile à réaliser et ils peuvent parfois mettre davantage l'accent sur l'une ou sur l'autre, faisant ainsi de ces tâches des activités opposées.

\section{Conclusion}

Malgré les défis ici soulignés, la formation pratique actuelle offerte en Colombie s'avère être une occasion précieuse pour aider les étudiants à comprendre les contextes éducatifs, sociaux et institutionnels de la profession enseignante. Il est à noter qu'à la fin de la formation pratique, la plupart des étudiants ont atteint les objectifs de formation visés et nous pouvons observer des progrès significatifs dans leur discours oral et écrit. Dans certains programmes, le mémoire de recherche permet aux étudiants de 


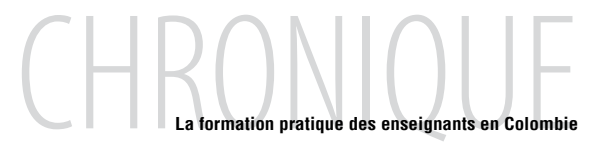

se plonger dans des sujets et des méthodologies spécifiques qui peuvent même parfois les motiver à poursuivre des études supérieures. La formation pratique facilite aussi la compréhension des éléments de base de l'interaction avec des élèves et des adultes, et ce, dans des contextes différents (urbains / ruraux, écoles publiques / écoles privées). Cette formation permet finalement aux étudiants de construire leurs propres représentations du rôle qui leur revient en tant que futurs enseignants dans un contexte pluriel et qui présente parfois des difficultés comme c'est les cas des conditions scolaires d'un pays comme la Colombie.

\section{Références}

Ministerio de Educación Nacional. (2014). Lineamientos de calidad para las licenciaturas en educación. Repéré à http://www.mineducacion.gov.co/cvn/1665/articles-340962 recurso 1.pdf

Republica de Colombia - Gobierno Nacional. (1994) Ley 115 de Febrero 8 de 1994. Repéré à

http://www.oei.es/quipu/colombia/Ley 115 1994.pdf 